Original Research Paper

\title{
Dynamical Analysis on the Transmission of Pertussis with Maternally Derived Immunity
}

\author{
Aisha Aliyu Yakubu, Farah Aini Abdullah, Ahmad Izani Md Ismail and Yazariah Mohd Yatim \\ School of Mathematical Sciences, Universiti Sains Malaysia, 11800 USM, Pulau Pinang, Malaysia
}

Article history

Received: 16-03-2020

Revised: 21-04-2020

Accepted: 20-06-2020

Corresponding Author:

Farah Aini Abdullah

School of Mathematical

Sciences, Universiti Sains

Malaysia, 11800 USM, Pulau

Pinang, Malaysia:

Email: farahaini@usm.my

\begin{abstract}
A susceptible-infected-recovered compartmental model incorporating maternally derived immunity compartment is analyzed in this study. The stability of the pertussis-disease-free and endemic equilibrium is studied. The basic reproduction number is obtained and its behavior analyzed by varying parameters. Numerical simulations indicated that when the waning parameter is increased, the frequency at which the population attains stability varies. However, the infected population does not go extinct even at equilibrium.
\end{abstract}

Keywords: Pertussis, Basic Reproduction Number, Stability Analysis, Numerical Simulations

\section{Introduction}

It was reported that globally, around 335 infectious diseases have emerged between 1940 to 2004 (Jones et al., 2008; Morens et al., 2004; Raslan et al., 2017). The World Health Organization (WHO) estimates that about one third of annual deaths worldwide are due to diseases spread by infection (WHO, 2015). Pertussis also known as "whooping" cough or the 100-days cough is an acute respiratory illness that has exhibited cyclical outbreaks over the last century (Auger et al., 2013). It is a highly contagious respiratory disease that can affect individuals of any age. Research indicates that infants $<1$ year old bear the largest disease burden (De Cellès et al., 2018; Fabricius et al., 2018; Tilahun et al., 2018). The disease is carried by a Gramnegative bacterium known as the Bordetella pertussis. The bacteria travels via respiratory droplets infecting human hosts (Koenig et al., 2019; Mattoo and Cherry, 2005). In the last two decades, there has been an increase in the incidence of the disease worldwide, with around 16 million cases occurring per year and approximately 200,000 deaths (Fabricius et al., 2018).

In this study, a mathematical model for the transmission of pertussis is studied. The model will be a modification of existing models (Anderson and May, 1982; Fabricius et al., 2013; Hu et al., 2014; Suleman and Riaz, 2019; Rozhnova and Nunes, 2012). These existing models considered population which consists of all age groups, where vaccination is introduced as a control for the disease to individuals that completely have no immunity. Thus, the modified model includes the maternally derived immunity compartment $(\mathrm{M})$ and the model will be in the form of a Maternally derived immunity Susceptible Infected Recovered (MSIR) model. This means that infants are born immune to the disease by gaining immunity from the mother or are given first dose of vaccine at birth. Thus, the model considers the transmission of the disease in infants as most literature have highlighted that the adverse effect of the disease occurs more within this group (Fabricius et al., 2018). The aim of this paper is to analyze and observe the dynamical behavior of pertussis model and to relate how certain proportions of the population behaves.

\section{Model Formulation}

In this section the model is formulated and analyzed. The total population of the model is partitioned into four compartments given by:

$$
N(t)=M(\mathrm{t})+S(\mathrm{t})+I_{w}(\mathrm{t})+R_{w}(\mathrm{t})
$$

The total population at the time $t$ denoted as $N(t)$ is made up of four compartments namely; maternally derived immunity $(M(t))$, susceptible $(S(t))$, infected $\left(I_{w}(t)\right)$ and recovered $\left(R_{w}(t)\right)$ compartments respectively and the subscript $(w)$ represents whooping cough. 


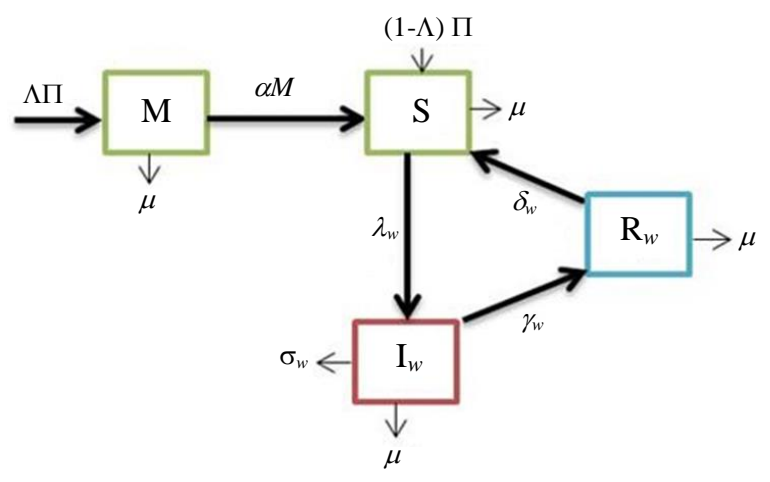

Fig. 1: Flow chart of MSIR model

In the model formulation, it is assumed that $\Lambda$ is the proportion of immunized individuals against infection, $\Pi$ is the per capita birth rate, the rate of vaccine efficiency is given by $\alpha$, the natural mortality rate is $\mu$, the force of infection is given by $\lambda_{w}=\beta_{w} c I_{w}$, which measures the per capita probability of acquiring the disease (Keeling and Rohani, 2011), the disease transmission rate is denoted by $\beta_{w}, c$ is the contact rate with an infected individual and $\delta_{w}$ is the progression rate due to loss of immunity, $\gamma_{w}$ is the recovery rate and $\sigma_{w}$ is the death rate caused by disease. Figure 1 above represents the flow chart of the MSIR model. The compartmental model for the disease transmission of pertussis is given by a system of nonlinear ordinary differential equation:

$$
\begin{aligned}
& \frac{d M}{d t}=\Lambda \Pi-(\alpha+\mu) M, \\
& \frac{d S}{d t}=(1-\Lambda) \Pi+\alpha M+\delta_{w} R_{w}-\left(\lambda_{w}+\mu\right) S, \\
& \frac{d I_{w}}{d t}=\lambda_{w} S-\left(\gamma_{w}+\sigma_{w}+\mu\right) I_{w}, \\
& \frac{d R_{w}}{d t}=\gamma_{w} I_{w}-\left(\delta_{w}+\mu\right) R_{w},
\end{aligned}
$$

where all the initial conditions are positive:

$$
M(0)=M_{0}>0, S(0)=S_{0}>0, I_{w}(0)=I_{w_{0}} \geq 0, R_{w}(0)=R_{w_{0}} \geq 0
$$

\section{Basic Properties of the Model}

In this next section, the basic properties of the model (2) is discussed. The model is shown to be positive and bounded in an invariant region. This analysis is very important when observing the dynamical behaviour of a disease model because it shows whether the model is epidemiologically appropriate and mathematically wellposed, that is the model and its predictions are certain (Mattoo and Cherry, 2005).

\section{Positivity of Solution}

\section{Theorem 1}

Let $\Omega$ be a positive invariant region and also suppose $\left\{\left(M(0)>0, S(0)>0, I_{w} \geq 0, R_{w} \geq 0\right)\right\} \in \Omega$, then all the solution set $\left(M(t), S(t), I_{w}(t), R_{w}(t)\right)>0$ of the model (2) are positive for subsequent time $(t)$ (Van Boven et al., 2002).

\section{Proof}

Given the equation from the susceptible compartment of the model (2):

$$
\begin{aligned}
& \frac{d S}{d t}=(1-\Lambda) \Pi+\alpha M+\delta_{w}-\left(\lambda_{w}+\mu\right) S \geq-\left(\lambda_{w}+\mu\right) S \\
& \frac{d S}{d t} \geq-\left(\lambda_{w}+\mu\right) S \\
& \int \frac{1}{S} d S \geq \int-\left(\lambda_{w}+\mu\right) d t \\
& S(\mathrm{t}) \geq S(0) \mathrm{e}^{-\left(\lambda_{w}+\mu\right) t} \\
& S(\mathrm{t}) \geq 0
\end{aligned}
$$

The third equation in the model (2) is also considered:

$$
\begin{aligned}
& \frac{d I_{w}}{d t}=\lambda_{w} S-\left(\gamma_{w}+\sigma_{w}+\mu\right) I_{w} \geq-\left(\gamma_{w}+\sigma_{w}+\mu\right) \\
& \frac{d I_{w}}{d t} \geq-\left(\gamma_{w}+\sigma_{w}+\mu\right) I_{w} \\
& \int \frac{1}{I_{w}} d I_{w} \geq \int-\left(\gamma_{w}+\sigma_{w}+\mu\right) d t \\
& I_{w}(\mathrm{t}) \geq I_{w}(0) \mathrm{e}^{-\left(\gamma_{w}+\sigma_{w}+\mu\right) t} \\
& I_{w}(\mathrm{t}) \geq 0
\end{aligned}
$$

It can also be shown for other compartments of the model (2) and as such the solution is positive for all $t>$ 0 . This completes the proof.

\section{The Positive Invariant Region}

This is the region that shows that the solution is bounded. The total population size for this model given by Equation (1) is differentiated and substituting respective expressions of, $\frac{d(M+S)}{d t}$ (Li and Guo, 2017), $\frac{d I_{w}}{d t}$ and $\frac{d R_{w}}{d t}$ yields:

$$
\begin{aligned}
\frac{d N}{d t} & =\frac{d(M+S)}{d t}+\frac{d I_{w}}{d t}+\frac{d R_{w}}{d t} \\
& =\Pi-\left(M+S+I_{w}+R_{w}\right) \mu-\sigma_{w} I_{w} \\
& =\Pi-\mu N-\sigma_{w} I_{w}
\end{aligned}
$$


The positive invariant region is obtained considering the following theorem (Bolarin and Omotola, 2016).

\section{Theorem 2}

The solutions of the model (2) are feasible for $t>0$ if they enter the invariant region $\Omega$.

\section{Proof}

Suppose $\Omega=\left(M, S, I_{w}, R_{w}\right)=R_{+}^{4}$ be any solution of the model (2) with non-zero initial conditions and there is no death due to disease $\left(\sigma_{w}=0\right)$, such that (3) can be written as:

$$
\begin{aligned}
& \frac{d N}{d t} \leq \Pi-\mu N \\
& \frac{d N}{d t}+\mu N \leq \Pi
\end{aligned}
$$

Solving (4) using the integrating factor yields:

$$
N(t)=\frac{\Pi}{\mu}+c e^{-\mu t}
$$

Given the initial condition $t=0$ and $N(0)=N_{0}$, then the solution becomes:

$$
N \leq \frac{\Pi}{\mu}+\left(N_{0}-\frac{\Pi}{\mu}\right) e^{-\mu t}
$$

Such that when $t \rightarrow \infty, N \rightarrow \frac{\Pi}{\mu}$.

Thus, the feasible solution set of the model (2) is epidemiologically meaningful and bounded in the domain:

$$
\Omega=\left\{\left(M, S, I_{w}, R_{w}\right) \in R_{+}^{4}: M, S, I_{w}, R_{w} \geq 0 ; N \leq \frac{\Pi}{\mu}\right\} .
$$

\section{Stability Analysis}

The stability analysis of the basic model (2) is to examine the behavior of the model having obtained the equilibrium points. This investigation establishes whether the equilibrium points are either asymptotically stable or unstable.

\section{Pertussis Disease-Free Equilibrium (PDFE)}

To obtain the disease-free equilibrium points in infectious disease modeling that is the state where there is no disease (pertussis) at the onset in the system, which implies that infected and recovered populations are zero $\left(I_{w}, R_{w}=0\right)$. The steady state of differential Equations (2) is obtained and the PDFE is given by (5), where $\phi=(1-$ $\Lambda) \Pi$, thus:

$$
E^{f}=\left(M^{f}, S^{f}, I_{w}^{f}, R_{w}^{f}\right)=\left(\frac{\Lambda \Pi}{\alpha+\mu}, \frac{\alpha \Lambda \Pi+(\alpha+\mu) \phi}{\mu(\alpha+\mu)}, 0,0\right) .
$$

\section{Basic Reproduction Number}

The basic reproduction number of the basic model (2) denoted $\mathfrak{R}_{0}$ is the average number of secondary infections produced by an index case of wholly susceptible population (Diekmann and Heesterbeek, 2000). It plays a critical role as a threshold parameter. The basic reproduction number $\mathfrak{R}_{0}=\rho\left(F V^{-1}\right)$, where $\rho$ is the spectral radius, $\mathrm{F}=\left[\beta_{w} c S\right]$ and $\mathrm{V}=\left[\gamma_{w}+\sigma_{w}+\mu\right]$. Thus $\mathfrak{R}_{0}$ is given by:

$\mathfrak{R}_{0}=\frac{\beta_{w} c(\alpha \Lambda \Pi+(\alpha+\mu) \phi)}{\mu(\alpha+\mu)\left(\gamma_{w}+\sigma_{w}+\mu\right)}$.

When $\mathfrak{R}_{0}<1$, it means that the probability of new cases of disease to persist in the population is insufficient for an outbreak to occur. If $\mathfrak{R}_{0}>1$, then the disease will become endemic causing a drastic decline in the population of the susceptible (Burrell et al., 2016).

Thus, the higher the value of $\mathfrak{R}_{0}$, the higher treatment coverage is required to eliminate the disease. From Equation (6), the behavior of $\mathfrak{R}_{0}$ in relation to the transmission rate $\left(\beta_{w}\right)$ is observed when the contact rate (c) of the model is varied. Figure 2 a reveals that when the contact rate of the disease in the population is high, the emergence of secondary infection increases and can become endemic. When the transmission of the disease is increased, as long as contact rate to the disease is kept minimal, the outbreak of the disease in the population is controlled and will not be endemic as observed in Fig. 2b.

\section{Local Stability of the Pertussis Disease-Free Equilibrium $\left(E^{f}\right)$}

To establish the local stability of the PDFE, the Jacobian of the model is considered. The characteristic equation is then derived from the Jacobian and thus the result of the eigenvalue is obtained.

\section{Theorem 3}

The PDFE of basic model (2) is locally asymptotically stable if $\mathfrak{R}_{0}<1$. 


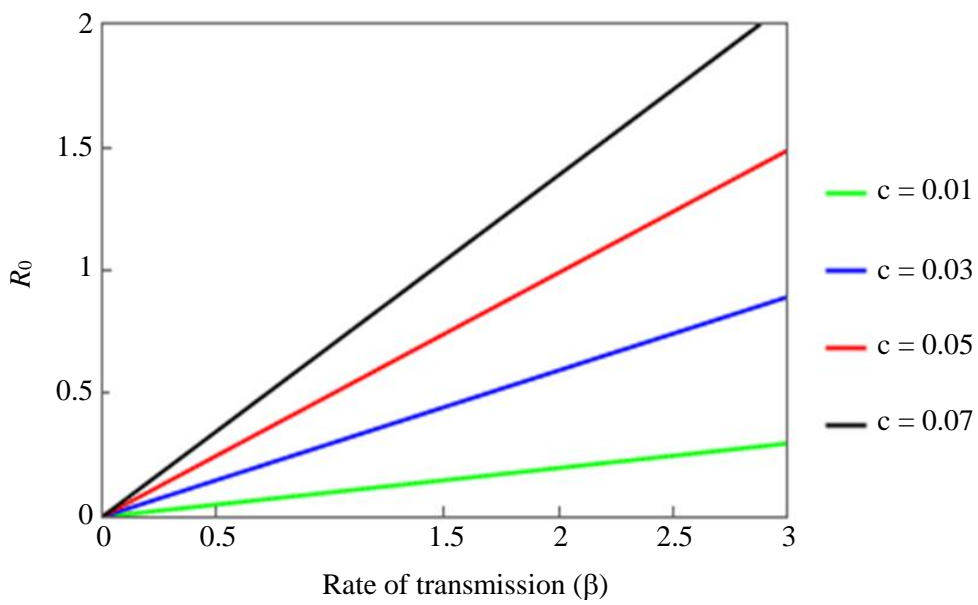

(a)

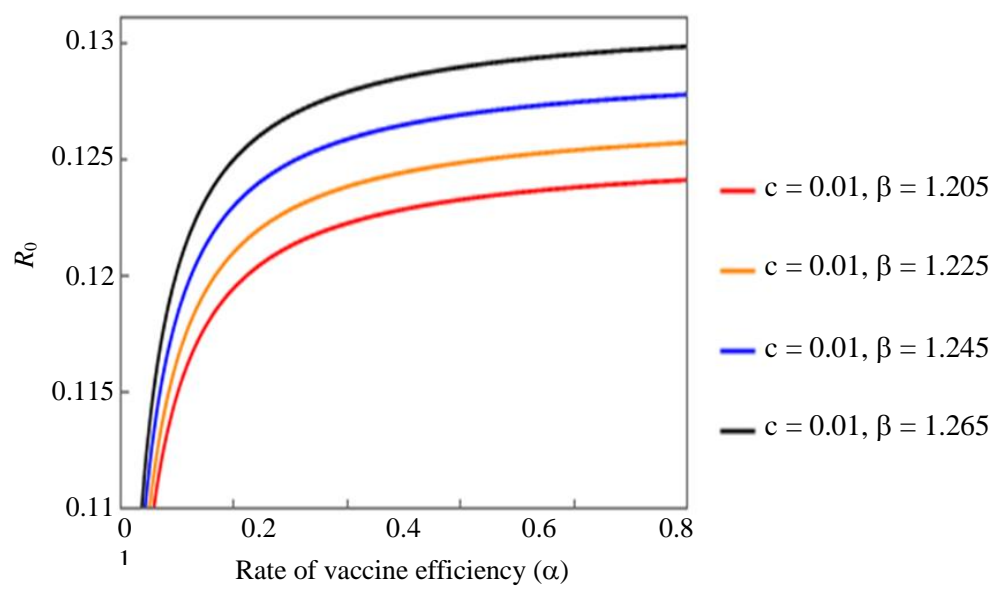

(b)

Fig. 2: The effect of contact rate, transmission rate and vaccine efficiency on the behavior of the basic reproduction number. (a) Variation of transmission rate at different values of contact rate $(c=0.01,0.03,0.05$ and 0.07$)$. (b) Variation of the vaccine efficiency at a fixed contact rate $(c=0.01)$ with different of transmission rates $\left(\beta_{w}=1.205,1.225,1.245\right.$ and 1.265) (Hu et al., 2014)

\section{Proof}

To establish the local stability of the system, the Jacobian of the basic model (2) is evaluated at PDFE which is given by:

$J\left(E^{f}\right)=\left(\begin{array}{cccc}-(\alpha+\mu) & 0 & 0 & 0 \\ \alpha & -\mu & \beta_{w} c S & 0 \\ 0 & 0 & \beta_{w} c S-\left(\gamma_{w}+\sigma_{w}+\mu\right) & 0 \\ 0 & 0 & \gamma_{w} & -\left(\delta_{w}+\mu\right)\end{array}\right)$ (7)

The characteristic equation of (7) is given by:

$(\lambda+\mu)(\lambda+\alpha+\mu)\left(\lambda+\delta_{w}+\mu\right)\left(\lambda+\gamma_{w}+\sigma_{w}+\mu-\beta_{w} c S\right)=0(8)$

It is clear from (8) that:

$$
\begin{aligned}
& \lambda_{1}=-\mu, \\
& \lambda_{2}=-(\alpha+\mu), \\
& \lambda_{3}=-\left(\mu+\delta_{w}\right), \\
& \lambda_{4}=-\left(\gamma_{w}+\sigma_{w}+\mu-\beta_{w} c S\right) .
\end{aligned}
$$

The analysis shows that all eigenvalues $\lambda_{1}, \lambda_{2}, \lambda_{3}, \lambda_{4}$ are negative provided $\alpha, \beta_{w}, \delta_{w}, c, \gamma_{w}, \mu$ and $\sigma_{w}$ are greater than zero. Therefore, considering the principle of linearized stability (Keeling and Rohani, 2011), the PDFE is asymptotically stable.

\section{Pertussis Endemic Equilibrium (PEE)}

The pertussis endemic equilibrium (PEE) is an equilibrium point that indicates that the disease will persist at steady state that is when $I_{w} \neq 0$. The PEE for the model (2) is given by $E^{e}=\left(M^{e}, S^{e}, I_{w}^{e}, R_{w}^{e}\right)$ : 


$$
\begin{aligned}
& M^{e}=\frac{\Lambda \Pi}{\alpha+\mu} \\
& S^{e}=\frac{\gamma_{w}+\sigma_{w}+\mu}{\beta_{w} c} \\
& I_{w}^{e}=\frac{\left(\delta_{w}+\mu\right)\left(c(\alpha \Lambda \Pi+(\alpha+\mu) \varphi) \beta_{w}-\mu(\alpha+\mu) Z\right)}{c(\alpha+\mu)\left(V\left(\delta_{w}+\mu\right)-\gamma_{w} \delta_{w}\right) \beta_{w}} \\
& R_{w}^{e}=\frac{\gamma_{w}\left(c(\alpha \Lambda \Pi+(\alpha+\mu) \varphi) \beta_{w}-\mu(\alpha+\mu) Z\right)}{c(\alpha+\mu)\left(V\left(\delta_{w}+\mu\right)-\gamma_{w} \delta_{w}\right) \beta_{w}}
\end{aligned}
$$

where, $Z=\left(\gamma_{w}+\sigma_{w}+\mu\right)$. It should be noted that the endemic equilibrium can only be feasible if $\mathfrak{R}_{0}>1$, which implies persistence of the disease.

Local stability of the pertussis endemic equilibrium $\left(E^{e}\right)$.

\section{Theorem 4}

The endemic equilibrium of the system (2) is locally asymptotically stable when $\mathfrak{R}_{0}>1$.

\section{Proof}

The Jacobian matrix is given by:

$$
J\left(E^{e}\right)=\left(\begin{array}{cccc}
-(\alpha+\mu) & 0 & 0 & 0 \\
\alpha & -\left(\beta_{w} c_{w}^{e}\right) & \beta_{w} c S & \delta_{w} \\
0 & \beta_{w} c I_{w}^{e} & \beta_{w} c S-\left(\gamma_{w}+\sigma_{w}+\mu\right) & 0 \\
0 & 0 & \gamma_{w} & -\left(\delta_{w}+\mu\right)
\end{array}\right)
$$

From (10), the characteristic equation written as:

$$
\lambda^{4}+A_{1} \lambda^{3}+A_{2} \lambda^{2}+A_{3} \lambda+A_{4}=0
$$

where, $A_{1}, A_{2}, A_{3}$ in (11) are the coefficients of $\lambda^{3}, \lambda^{2}, \lambda$ and $A_{4}$ the constant term are given by:

$$
\begin{aligned}
& A_{1}=\left(\alpha+3 \mu+Z+c\left(\mathrm{I}_{w}^{e}-S^{e}\right) \beta_{w}+\delta_{w}\right) \\
& A_{2}=\left(\begin{array}{l}
3 \mu(\mu+Z)+\alpha(2 \mu+Z)-2 c^{2} S^{e} \mathrm{I}_{w}^{e} \beta_{w}^{2}+(\alpha+2 \mu+Z) \delta_{w} \\
+\beta_{w} c\left(-S^{e}\left(\alpha+3 \mu+\delta_{w}\right)+\mathrm{I}_{w}^{e}\left(\alpha+2 \mu+Z+\delta_{w}\right)\right)
\end{array}\right) \\
& A_{3}=\left(\begin{array}{l}
\mu\left(\alpha \mu+\mu^{2}+2 \alpha \mathrm{Z}+3 \mu Z\right)+(\mu(\alpha+\mu)+(\alpha+2 \mu) \mathrm{Z}) \delta_{w} \\
-2 c^{2} S^{e} \mathrm{I}_{w}^{e} \beta_{w}^{2}\left(\alpha+2 \mu+\delta_{w}\right)+\beta_{w} c\left(-S^{e}\left(\mu(2 \alpha+3 \mu)+(\alpha+2 \mu) \delta_{w}\right)\right. \\
\left.+\mathrm{I}_{w}^{e}\left(\mu(\alpha+\mu)+(\alpha+2 \mu) Z+\left(\alpha+\mu+\mathrm{Z}-\gamma_{w}\right) \delta_{w}\right)\right)
\end{array}\right) \\
& A_{4}=\left(\begin{array}{l}
\left.(\alpha+\mu)\left(\begin{array}{l}
\mu Z\left(\mu+\delta_{w}\right)-2 c^{2} S^{e} \mathrm{I}_{w}^{e} \beta_{w}^{2}\left(\mu+\delta_{w}\right) \\
-\beta_{w} c\left(S ^ { e } \mu \left(\mu\left(2 \alpha+\delta_{w}\right)-\mathrm{I}_{w}^{e}\left(\mu Z+\left(Z-\gamma_{w}\right) \delta_{w}\right)\right.\right.
\end{array}\right)\right)
\end{array}\right.
\end{aligned}
$$

Considering the Routh-Hurwitz criterion, for $\mathfrak{R}_{0}>1$, the endemic equilibrium $\left(E^{e}\right)$ is asymptotically stable if:

$$
\begin{aligned}
& A_{1}>0, A_{2}>0, A_{3}>0, A_{4}>0 ; A_{1} A_{3}-A_{3}>0 \text { and } \\
& A_{1} A_{3}-A_{3}>0 .
\end{aligned}
$$

\section{Numerical Simulation}

In this section, the numerical simulation of the model (2) is carried out to demonstrate its asymptotic behavior. The initial condition of the state variables is given by $M\left(t_{0}\right)=0.304, S\left(t_{0}\right)=0.604$ and $I_{w}\left(t_{0}\right)=R_{w}\left(t_{0}\right)=0$ at the disease-free state and $I_{w}\left(t_{0}\right)=0.4, R_{w}\left(t_{0}\right)=0.3$ at an endemic state from the initial time $t_{0}$ to the unit time $\left(t_{f l}\right.$ $=60$ days) (Edwards, 2005; Wang et al., 2019). Figure 3 gives a time series graph which illustrates the behavior of population with respect to time.

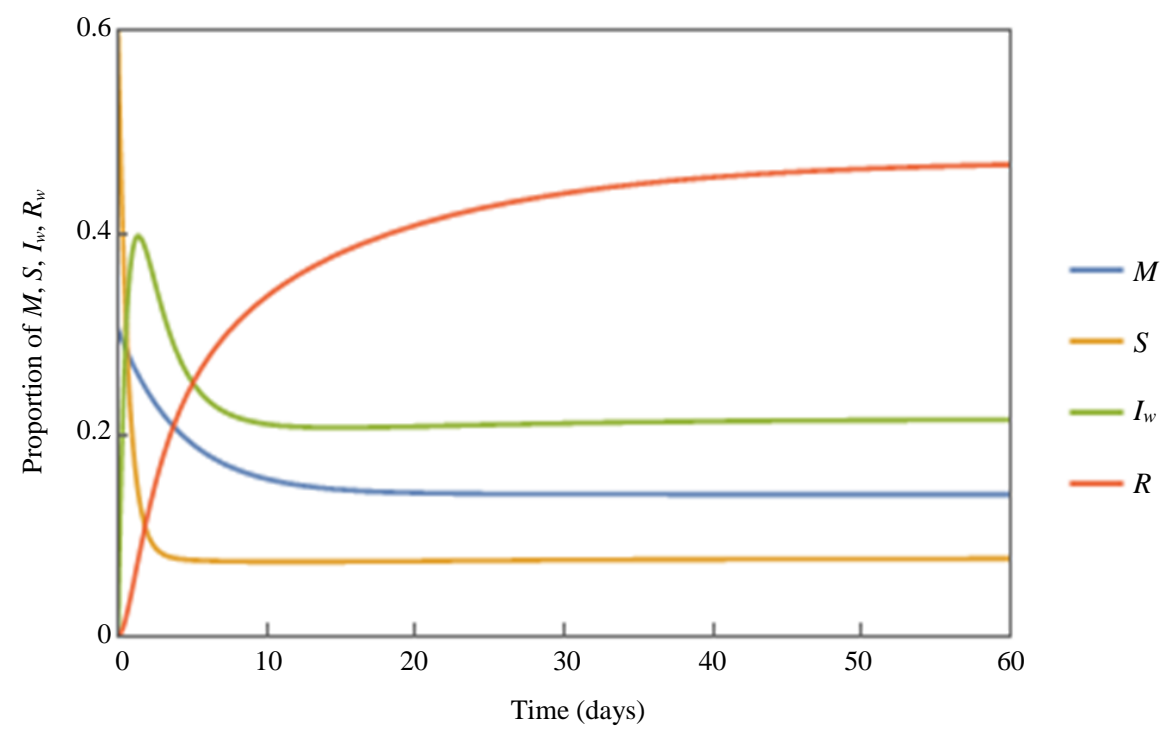


Aisha Aliyu Yakubu et al. / Journal of Mathematics and Statistics 2020, Volume 16: 104.112 DOI: $10.3844 / j m s s p .2020 .104 .112$

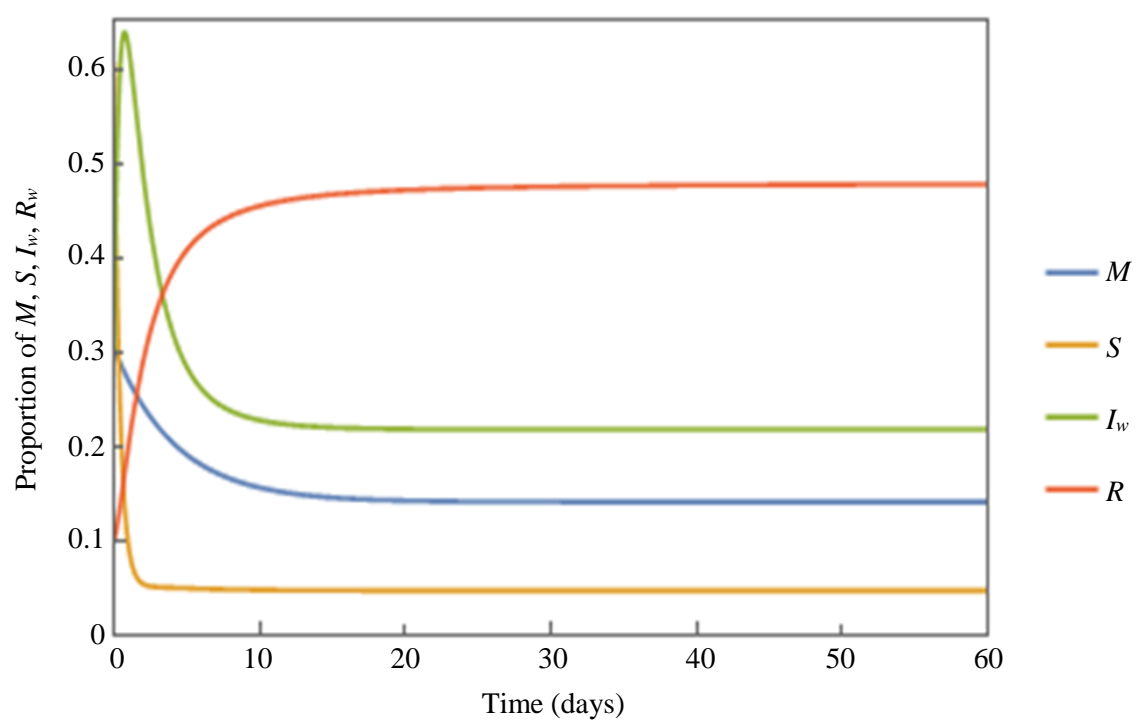

(b)

Fig. 3: Time series plot at (a) pertussis disease-free and (b) endemic state

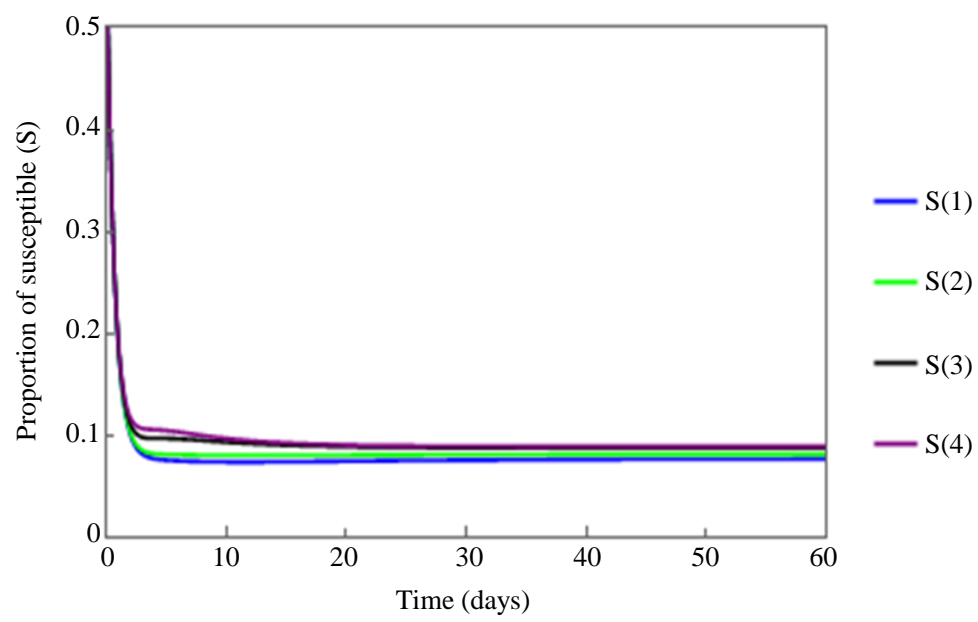

(a)

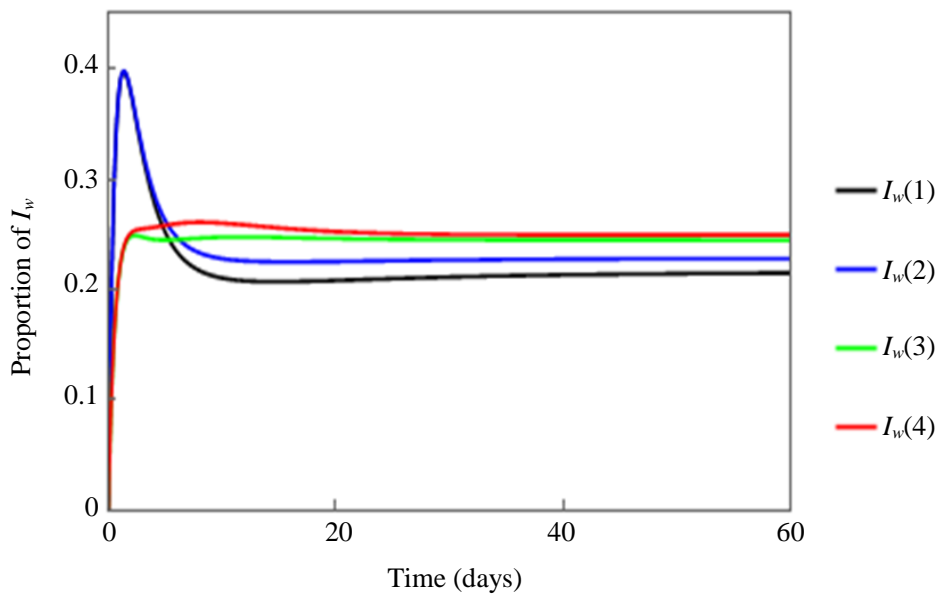

(b) 
Aisha Aliyu Yakubu et al. / Journal of Mathematics and Statistics 2020, Volume 16: 104.112 DOI: 10.3844/jmssp.2020.104.112

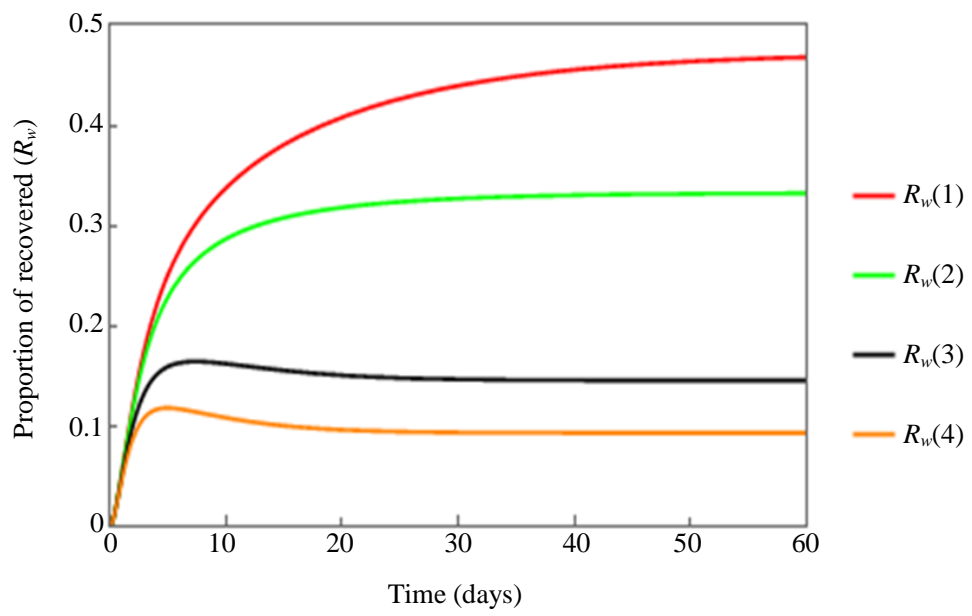

(c)

Fig. 4: Effects of varying the immunity waning parameter $\left(\delta_{w}=0.06,0.106,0.306,0.506\right)$ the pertussis disease-free state; (a) Susceptible; (b) Infected; (c) Recovered

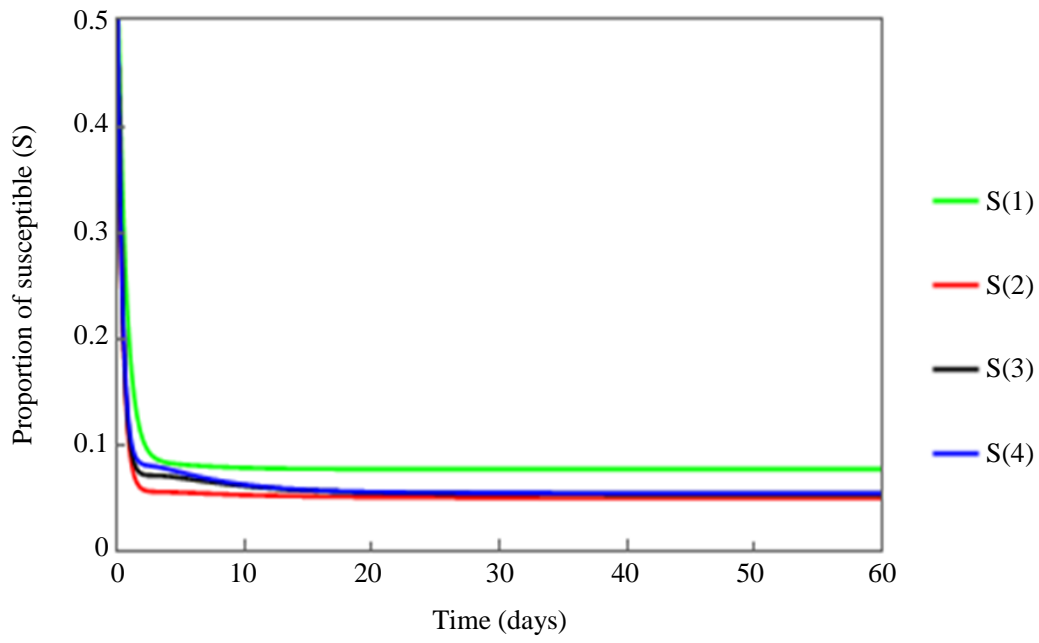

(a)

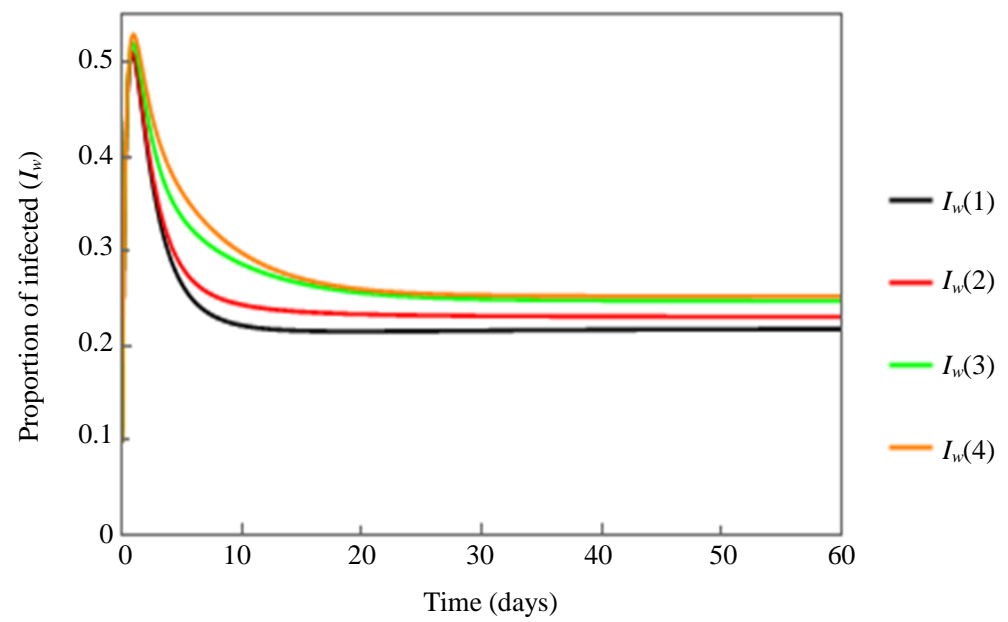

(b) 


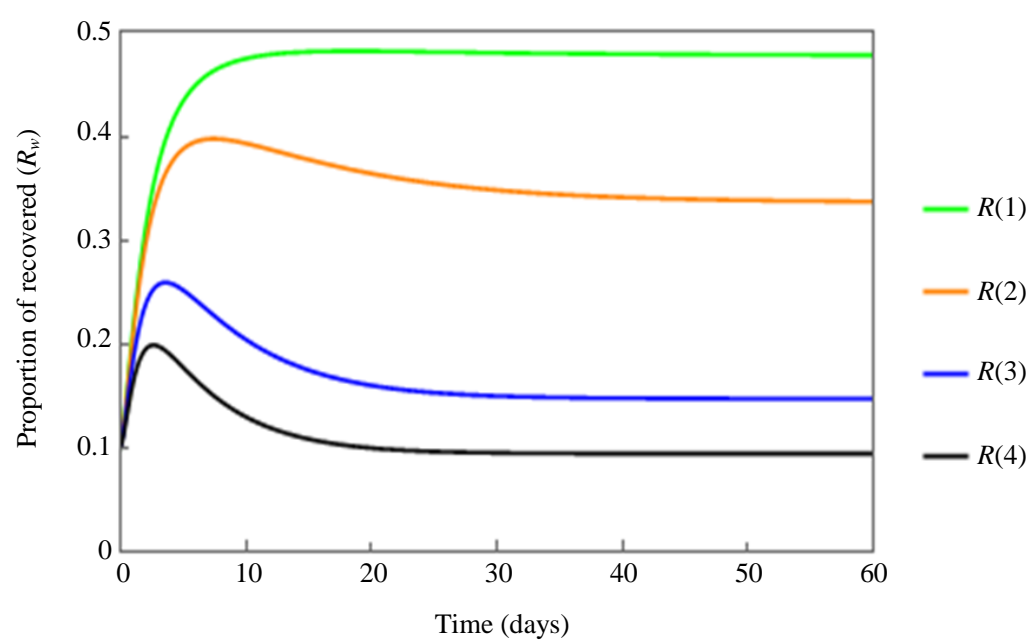

(c)

Fig 5: Effects of varying the immunity waning parameter $\left(\delta_{w}=0.06,0.106,0.306,0.506\right)$ on the population at an endemic state; (a) Susceptible; (b) Infected; (c) Recovered

The time series plot in Fig. 3b shows a difference in the steepness of the infected population as compared to Fig. 3a. However, none of the states exhibits total extinction of the disease. The maternally derived population decreases and moves to the susceptible compartment. The next illustration, Fig. 4 shows the behavior of each population with variation in some parameters that affect the system. The numerical simulation in Fig. 4a shows a decrease in the susceptible population. This indicates that as a result of immunity waning, the individuals in this group move to the infected population.

However, not all individuals are affected with the disease as the point of equilibrium shows that certain proportion of the individuals are not infected. In Fig. 4b, the simulation reveals that with an increase in the immunity waning parameter $\delta_{w}$ the spread of infection increases in the population. Although the population does not go extinct, it approaches the equilibrium state at different population sizes.

The recovered population as observed in Fig. $4 \mathrm{c}$ tends to vary in sizes in a descending order and thus individuals become susceptible again which makes them prone to infection. The characteristic of population as observed in Fig. $5 \mathrm{a}$ and $5 \mathrm{c}$ in an endemic state is similar to the case of the disease free with variation in the immunity waning parameter, but the size of infected individual varies. Figure $5 b$ shows that at all stages of the immunity waning, the infection spread is quite high and still does not go extinct.

\section{Discussion}

In this study, an MSIR model was used to study the transmission dynamics of Pertussis disease in infants. The model was divided into the total population of four compartments; maternally derived immunity, susceptible, infected and recovered. From the analysis, it was seen that the model exists in an invariant region making it epidemiologically appropriate and mathematically wellposed. The behavior of $\mathfrak{R}_{0}$ was observed, where it showed that an increase in the contact rate will lead to the emergence of secondary infection and this can become endemic. The local stability of the model was derived using the Jacobian and Routh-Hurtwiz criterion for both disease-free and endemic states respectively the result showed that the disease-free and endemic equilibrium are both asymptotically stable. Also from Theorems 3 and 4 , the behavior of the basic reproduction was used to establish the stability of the system, which showed that when $\mathfrak{R}_{0}<1$ the PDEF is stable and when $\mathfrak{R}_{0}>1$ the PEE is stable, otherwise unstable.

Numerical simulations were conducted and the behavior of the individuals in various compartments was observed. The result shows that when there is variation in the immunity waning parameter the infected population in the endemic state seems quite high. However, for both cases of PDFE and PEE, the population of the infected individuals never goes extinct. Biologically, it means the disease might persist if adequate measures are not put in place. Furthermore, it will be appropriate to investigate the dynamical behavior of model (2) with the inclusion of vaccine as a treatment. This further investigation might give an insight why there is reemergence of the disease despite pertussis being a vaccine preventable disease.

\section{Acknowledgement}

This research was supported by the Bridging Grant Scheme,304/PMATHS/6316285 from Research Creativity and Management Office (RCMO), Universiti Sains Malaysia. The authors would also like to thanks School of 
Mathematical Sciences, Universiti Sains Malaysia for providing computing and research assistance.

\section{Author's Contributions}

All authors contributed immensely to this work.

\section{Ethics}

This article is original and contains unpublished materials. Thus, there is no ethical issue involved in this manuscript.

\section{References}

Anderson, R.M. and R.M. May, 1982. Directly transmitted infectious diseases: Control by vaccination. Science, 215: 1053-1060.

DOI: $10.1126 /$ science.7063839

Auger, K.A., S.W. Patrick and M.M. Davis, 2013. Infant hospitalizations for pertussis before and after Tdap recommendations for adolescents. Pediatrics, 132: e1149-e1155. DOI: 10.1542/peds.2013-1747

Bolarin, G. and I.U. Omatola, 2016. A mathematical analysis of HIV/TB co-infection model. Applied Math., 6: 65-72. DOI: 10.5923/j.am.20160604.01

Burrell, C.J., C.R. Howard and F.A. Murphy, 2016. Fenner and White's Medical Virology. 5th Edn., Academic Press, ISBN-10: 9780123751560, pp: 604.

De Cellès, M.D., F.M. Magpantay, A.A. King and P. Rohani, 2018. The impact of past vaccination coverage and immunity on pertussis resurgence. Sci. Translat. Med., 10: 1748-1748.

DOI: $10.1126 /$ scitranslmed.aaj 1748

Diekmann, O. and J.A.P. Heesterbeek, 2000. Mathematical Epidemiology of Infectious Diseases: Model Building, Analysis and Interpretation. 1st Edn., John Wiley and Sons, ISBN-10: 0471986828, pp: 303.

Edwards, K.M., 2005. Overview of pertussis: Focus on epidemiology, sources of infection and long term protection after infant vaccination. Pediatric Infect. Dis. J., 24: S104-S108.

DOI: $10.1097 / 01 . i n f .0000166154 .47013 .47$

Fabricius, G., P.E. Bergero, M.E. Ormazabal, A.L. Maltz and D.F. Hozbor, 2013. Modelling pertussis transmission to evaluate the effectiveness of an adolescent booster in Argentina. Epidemiol. Infect., 141: 718-734. DOI: 10.1017/S0950268812001380

Fabricius, G., P.M. Aispuro, P. Bergero, D. Bottero and M. Gabrielli et al., 2018. Pertussis epidemiology in Argentina: TRENDS after the introduction of maternal immunisation. Epidemiol. Infect., 146: 858-866. DOI: 10.1017/S0950268818000808

$\mathrm{Hu}, \mathrm{X}$., Y. Zhang and F. Sun, 2014. Threshold dynamics for a pertussis model with seasonality. Int. J. Nonlinear Sci., 17: 281-288.
Jones, K.E., N.G. Patel, M.A. Levy, A. Storeygard and D. Balk et al., 2008. Global trends in emerging infectious diseases. Nature, 451: 990-993. DOI: $10.1038 /$ nature06536

Keeling, M.J. and P. Rohani, 2011. Modeling Infectious Diseases in Humans and Animals. 1st Edn., Princeton University Press, ISBN-10: 1400841038, pp: 408.

Koenig, K.L., J. Farah, E.C. McDonald, S. Thihalolipavan and M.J. Burns, 2019. Pertussis: The identify, isolate, inform tool applied to a re-emerging respiratory illness. Western J. Emergency Med., 20: 191-191. DOI: $10.5811 /$ westjem.2018.11.40023

Li, H. and S. Guo, 2017. Dynamics of a SIRC epidemiological model. Electron. J. Differential Eq., 2017: 1-18.

Mattoo, S. and J.D. Cherry, 2005. Molecular pathogenesis, epidemiology and clinical manifestations of respiratory infections due to Bordetella pertussis and other Bordetella subspecies. Clin. Microbiol. Rev., 18: 326-382. DOI: 10.1128/CMR.18.2.326-382.2005

Morens, D.M., G.K. Folkers and A.S. Fauci, 2004. The challenge of emerging and re-emerging infectious diseases. Nature, 430: 242-249.

DOI: $10.1038 /$ nature02759

Raslan, R., S. El Sayegh, S. Chams, N. Chams and A. Leone et al., 2017. Re-emerging vaccinepreventable diseases in war-affected peoples of the eastern Mediterranean region-an update. Frontiers Public Health, 5: 283-283.

Rozhnova, G. and A. Nunes, 2012. Modelling the long-term dynamics of pre-vaccination pertussis. J. Royal Society Interface, 9: 2959-2970. DOI: 10.1098/rsif.2012.0432

Suleman, M. and S. Riaz, 2019. An optimal control of vaccination applied to whooping cough model. J. Math., 51: 121-136.

Tilahun, G.T., O.D. Makinde and D. Malonza, 2018. Codynamics of pneumonia and typhoid fever diseases with cost effective optimal control analysis. Applied Math. Comput., 316: 438-459.

DOI: 10.1016/j.amc.2017.07.063

Van Boven, M., H.E. de Melker, J.F. Schellekens and M. Kretzschmar, 2000. Waning immunity and subclinical infection in an epidemic model: Implications for pertussis in The Netherlands. Math. Biosci., 164: 161-182.

Wang, X., Y. Shi, J. Cui and Z. Feng, 2019. Analysis of age-structured pertussis models with multiple infections during a lifetime. J. Dynam. Differential Eq., 31: 2145-2163.

WHO, 2015. Weekly epidemiological record Relevé épidémiologique hebdomadaire. World Health Organization. 